\title{
Prevalência de obesidade em adultos e seus fatores de risco
}

\section{Prevalence and risk factors of obesity in adults}

\section{Denise P. G igante, Fernando C. Barros, Cora L.A. Post e Maria T.A. 0 linto}

Departamento de Nutrição da U niversidade Federal de Pelotas. Pelotas, RS - Brasil (D.P.G., C.L.A.P.); D epartamento de Medicina Social da U niversidade Federal de Pelotas. Pelotas, RS - Brasil (F.C.B.); Centro de Pesquisas Epidemiológicas da U niversidade Federal de Pelotas. Pelotas, RS - Brasil (M.T.A.O.) 


\title{
Prevalência de obesidade em adultos e seus fatores de risco*
}

\section{Prevalence and risk factors of obesity in adults}

\author{
Denise P. Gigante, Fernando C. Barros, Cora L.A. Post e Maria T.A. 0 linto
}

Departamento de Nutrição da U niversidade Federal de Pelotas. Pelotas, RS - Brasil (D.P.G., C.L.A.P.); D epartamento de Medicina Social da U niversidade Federal de Pelotas. Pelotas, RS Brasil (F.C.B.); Centro de Pesquisas Epidemiológicas da U niversidade Federal de Pelotas.

Pelotas, RS - Brasil (M.T.A.O.)

Material e Método

Resultados

Conclusão

\section{Resumo}

Introdução

Foi realizado estudo transversal em uma amostra representativa da população adulta de Pelotas para determinar a prevalência de obesidade e os fatores a ela associados, tendo em vista o acentuado aumento de excesso de peso no Brasil, entre 1974 e 1989.

Foram estudadas 1.035 pessoas com idade entre 20 e 69 anos, residentes na zona urbana do município. A obesidade foi definida a partir do índice de massa corporal (IMC) igual ou superior a $30 \mathrm{~kg} / \mathrm{m}^{2}$. A análise multivariada foi realizada considerando um modelo hierárquico das variáveis associadas com obesidade em ambos os sexos.

A prevalência de obesidade foi de $21 \%$ ( IC $_{95 \%} 18$ - 23), sendo de $25 \%$ ( IC $_{95 \%} 22$ - 29) entre as mulheres e $15 \%$ ( $\mathrm{IC}_{95 \%} 12$ - 18) entre os homens. A relação entre as variáveis socioeconômicas e a obesidade foi inversa entre as mulheres e direta entre os homens. Entre as mulheres, as variáveis que se mantiveram associadas significativamente com obesidade foram: obesidade dos pais, ocorrência de diabete ou hipertensão, não fumar, menor número de refeições diárias e não ter realizado exercício físico no lazer durante o último ano. Para os homens somente a ocorrência de obesidade nos pais e a hipertensão arterial sistêmica estiveram significativamente associadas, enquanto a proteção do maior número de refeições apresentou uma associação quase significativa $(p=0,07)$.

Os resultados indicam que os determinantes de obesidade são diferentes entre os sexos, ocorrendo em maior frequiência entre as mulheres e com o aumento da idade.

Obesidade, epidemiologia. Fatores de risco.

\footnotetext{
* Parte de dissertação de mestrado de autoria de D. P. Gigante, apresentada ao Departamento de Nutrição Social da Universidade Federal de Pelotas. Financiado pela Fundação de Amparo à Pesquisa do Rio Grande do Sul (Processo no 93/2955-1).

Correspondência para/Correspondence to: Denise P. Gigante - Departamento de Nutrição da Universidade Federal de Pelotas. Caixa Postal 354 96010-900 Pelotas, RS - Brasil. E mail: denise@ufpel.tche.br

Recebido em 29.5.1996. Aprovado em 25.11.1996.
} 


\begin{abstract}
Introduction

Material and Methods

A population-based cross-sectional study was conducted in Pelotas, Southern Brazil, with the objective of determining the prevalence of obesity and identify associated, variables as this condition increased markedly in the country between 1974 and 1989.

One thousand and thirty-five adults between 20 and 69 years of age were studied. Obesity was defined as a Body Mass Index - BMI - equal to or over $30 \mathrm{Kg} /$ square meter). The multivariate analyses took into account the hierarchical model of the variables associated with obesity for both men and women.

Results The prevalence for the overall population was of $21 \%$ (CI 18 - 23). It was higher among women - 25\% (CI 22 - 29) than for men - 15\% (CI 12 - 18). Socioeconomic status was positively associated with obesity among men, whereas the opposite situation was reported for women, with those belonging to the poorest social strata presenting increased BMI. Reported obesity in their parents was associated with increased BMI in the subjects, and this association remained statistically significant even after compensating for the effect of possible confounding variables. Self-reported diabetes and arterial hypertension doubled the risk of obesity, whereas non-smoking was associated with obesity only among women. Variables which were not associated with obesity after adjusting for confounders were alcohol consumption, marital status and parity. Women having more daily meals were less prone to obesity, even after controlling for confounders, and this association was not quite significant for men $(p=0.07)$.
\end{abstract}

Conclusions The prevalence of obesity was higher among women, and important differences in risk factors were noticed when the population was considered by sex.

Obesity, epidemiology. Risk factors.

\section{INTRO DUÇÃO}

As prevalências de obesidade em países desenvolvidos, como Suécia ${ }^{14}$ e Estados Unidos ${ }^{13}$, têm aumentado nas últimas décadas. Uma revisão da literatura ${ }^{28}$ mostra que, nesses países, a melhoria da condição socioeconômica está associada com um decréscimo na prevalência de obesidade nas mulheres. Por outro lado, nessas sociedades a relação entre o nível socioeconômico e a obesidade em homens é complexa e pouco compreendida.

No Brasil, entre 1974 e $1989^{8}$, a proporção de pessoas com excesso de peso aumentou de $21 \%$ para $32 \%$. Dentre as regiões do País, o Sul apresenta as maiores prevalências de obesidade, sendo essas semelhantes e, até mesmo superiores, a países desenvolvidos. A evolução da ocorrência de obesidade nesse período, em relação ao sexo, dobrou entre os homens (de $2,4 \%$ para $4,8 \%$ ), enquanto que entre a população feminina o aumento da obesidade também foi significativo (7\% para $12 \%)$. Os determinantes da maior freqüência de obesidade entre as mulheres são ainda desconhecidos.
No que se refere à situação socioeconômica, os resultados da Pesquisa Nacional de Saúde e Nutrição (PNSN) ${ }^{8}$, para o País como um todo, mostraram que a prevalência de excesso de peso aumenta de acordo com o poder aquisitivo, especialmente entre os homens. Em um estudo sobre fatores de risco para doenças crônicas realizado em Porto Alegre ${ }^{9}$, observou-se que a obesidade afeta principalmente os homens de classes sociais mais altas e as mulheres de menor nível socioeconômico.

As conseqüências do excesso de peso à saúde têm sido demonstradas em diversos trabalhos ${ }^{4,20,30}$. A obesidade é fator de risco para hipertensão arterial ${ }^{25}$, hipercolesterolemia ${ }^{18}$, diabetes mellitus ${ }^{18}$, doenças cardiovasculares ${ }^{19} \mathrm{e}$ algumas formas de câncer ${ }^{10}$.

Em Pelotas, a obesidade foi estudada como fator de risco para hipertensão arterial $^{21}$, e os obesos mostraram um risco 2,5 vezes maior de apresentarem hipertensão, quando comparados aos indivíduos de peso adequado.

Por estar a obesidade aumentando no País, e sendo esta condição um fator de risco para patologias importantes, decidiu-se realizar a presente investi- 
gação para determinar sua prevalência e os fatores que a determinam, ou estão com ela associados, em uma população adulta do Município de Pelotas. O presente estudo aborda a prevalência da obesidade, e sua relação com sexo, fatores socioeconômicos, demográficos e culturais, morbidades referidas e variáveis de alimentação.

\section{MATERIAL E MÉTO DO}

Realizou-se um estudo de delineamento transversal, de base populacional, onde a população a ser avaliada foi escolhida através de um processo amostral aleatório em estágios múltiplos ${ }^{3}$ entre pessoas com idades entre 20 e 69 anos, residentes na zona urbana do Município de Pelotas, RS.

O cálculo do tamanho da amostra baseou-se em estudo anterior ${ }^{21}$ que estimou a prevalência de obesidade em $20 \%$. Para o estudo da prevalência de obesidade considerou-se aceitável uma margem de erro de $2,5 \%$ e nível de confiança de $95 \%$. Para estudar a associação com os fatores de risco, considerou-se o sedentarismo como a exposição de maior freqüência, com uma razão de 4 expostos para cada não exposto. Com um poder de $80 \%$, uma razão de prevalências de 2,0 e uma prevalência de doença de $10 \%$ para os não expostos, obteve-se 720 indivíduos. A esse número foram acrescidos $10 \%$ para possíveis perdas e $30 \%$ para análise estratificada.

Para obter-se o número de pessoas suficiente foi necessário visitar 600 domicílios, baseado em informações do censo do $\mathrm{IBGE}^{7}$ de 1991, que indicou uma média de 1,8 pessoas entre 20-69 anos de idade por domicílio, no Município de Pelotas.

Com base nesses dados foram sorteados, sistematicamente, 30 setores censitários onde seria realizado o estudo. Também aleatoriamente, foi sorteada, em cada setor, a esquina por onde o estudo deveria iniciar e a partir dessa, sistematicamente, as vinte casas a serem visitadas. Em cada um dos domicílios selecionados foram entrevistados todos os moradores com idade entre 20 e 69 anos, através de um questionário abordando fatores biológicos, socioeconômicos, familiares, antropométricos, de consumo alimentar e de atividade física.

A obesidade foi definida a partir do índice de massa corporal(IMC) que é obtido pela divisão da massa corporal (em quilogramas) pela estatura (em metros ao quadrado), sendo esse um indicador apropriado para avaliação do estado nutricional de adultos ${ }^{1}$. Foram consideradas obesas as pessoas que apresentaram IMC igual ou superior a $30 \mathrm{~kg} / \mathrm{m}^{2}$ e com sobrepeso aquelas com IMC entre 25 e $30 \mathrm{~kg} / \mathrm{m}^{2}$, de acordo com a Organização Mundial de Saúde ${ }^{31}$.

Foram estudadas as seguintes variáveis: sexo; cor da pele; idade (em anos completos); situação conjugal (entrevistados que viviam com ou sem companheiro na época do estudo); paridade (número total de filhos de cada uma das mulheres entrevistadas - nascidos vivos ou mortos); escolaridade (em anos completos cursados na escola); renda familiar (renda mensal em salários mínimos de todos os moradores do domicílio, distribuída por quartis 0 a $2,73,2,74$ a $4,77,4,78$ a 8,88 e 8,89 a 80 saláriosmínimos); classe social de Bronfman ${ }^{17}$ (construída a partir da escolaridade, renda e ocupação do chefe da família, distribuída em burguesia, nova pequena burguesia, pequena burguesia tradicional, proletariado não típico, proletariado típico e subproletariado); classe social proposta pela Associação Brasileira de Institutos de Mercado Abipeme $^{24}$ (construída a partir de bens e utilidades domésticas, além da escolaridade, distribuída por classes A, B, C, D e E de acordo com escore estabelecido); trabalho remunerado realizado no mês anterior à entrevista; exercício físico no lazer realizado durante o último ano; natureza da atividade (opinião do entrevistado sobre sua atividade física diária, distribuída em leve, moderada ou pesada); número de refeições diárias (foram consideradas como refeições, além das habituais - café da manhã, almoço e jantar - outras que possam ter sido realizadas durante a manhã, à tarde ou à noite); consumo de açúcar (construído a partir do consumo mensal da família distribuído pelo número de pessoas que participam do grupo de consumo familiar); consumo de gordura (construído a partir da quantidade de óleo consumida pela família no último mês distribuída pelo número de pessoas que participam do grupo de consumo familiar); consumo de álcool (consumo de diferentes tipos de bebidas alcóolicas cerveja, vinho, cachaça, whisky, vodka, outros - no último mês); tabagismo (distribuído em três categorias, sendo considerados fumantes aqueles que fumavam pelo menos 1 cigarro por dia); hipertensão arterial sistêmica e diabetes mellitus (referidas pelo entrevistado); obesidade dos pais (referida pelo entrevistado através da pergunta se seu pai/mãe é ou era gordo/gorda); massa corporal (através da utilização de balança de banheiro marca "Bender" com capacidade de $150 \mathrm{~kg}$ e precisão de $0,5 \mathrm{~kg}$ ); estatura (coletada uma única vez com um antropômetro de alumínio construído exclusivamente para pesquisa de campo com uma base móvel formando um ângulo de $90^{\circ}$ com a régua com precisão de $0,1 \mathrm{~cm}$ ).

O trabalho de campo foi realizado entre janeiro e maio de 1994 com a participação de 22 entrevistadores, estudantes das Faculdades de Nutrição e Medicina, que trabalharam em duplas, com a finalidade de obter medidas antropométricas mais fidedignas.

Os entrevistadores foram submetidos a um treinamento para aplicação do questionário e padronização da tomada de medidas antropométricas, segundo a recomendação de Lohman e col $^{16}$. Durante esta coleta os entrevistados estavam descalços e usando roupas leves.

O controle de qualidade foi realizado através de revisita em 5\% dos domicílios, quando algumas informações foram conferidas.

As análises uni e bivariadas foram realizadas com os programas SPSS/PC+ e Epi Info, utilizando como medida de efeito a razão de prevalências e seus intervalos de confiança. 


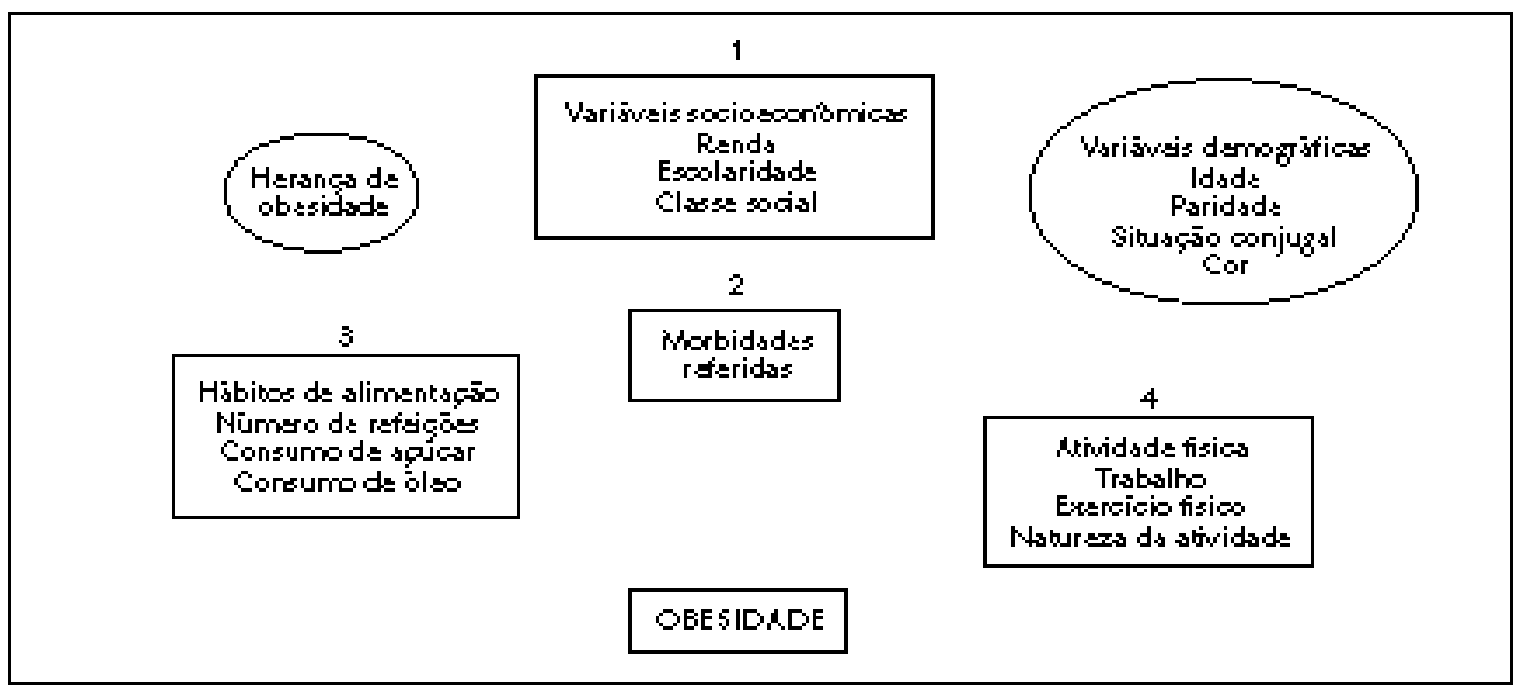

Figura 1 - Modelo hierárquico das relações entre os fatores de risco para obesidade.

Figure 1 - Hierarquical model of risk factors for obesity.

Foram feitas análises bi e multivariada para homens e mulheres através de regressão logística, com o programa EGRET, a partir de um modelo hierárquico (Figura 1). Utilizou-se razão de odds como medida de efeito, sabendo-se que esta é ligeiramente superior à razão de prevalência, quando fator de risco, e inferior, quando fator de proteção.

De acordo com o modelo (Figura 1), foram analisadas as variáveis de cada bloco, hierarquicamente, ajustadas para aquelas que se encontravam no nível imediatamente superior, sendo mantidas no modelo as variáveis que apresentavam significância estatística $(p<0,1)$ através do teste de razão de verossimilhanças (TRV). Na análise dos possíveis fatores de confusão utilizou-se esse nível de significância para evitar a exclusão de variáveis potencialmente importantes. Desta forma alguns dos intervalos de confiança de $95 \%$ poderão incluir a unidade. As variáveis categóricas foram incluídas na análise como ordinais quando o teste para tendência linear foi significativo, sem desvio da linearidade.

\section{RESULTADOS}

Foram visitados 600 domicílios, dos quais em seis não foi possível contactar os moradores. Das 1.145 pessoas com idade entre 20 e 69 anos, encontradas nos domicílios contactados, foram entrevistadas 1.035, tendo ocorrido $5,9 \%$ de recusas e $3,8 \%$ de perdas.

Metade da amostra encontrava-se na faixa entre 20 e 40 anos, sendo que as mulheres constituíram 56\% da população estudada. Houve predomínio de pessoas de cor branca (86\%) e casadas (68\%). Quanto à situação socioeconômica, aproximadamente um terço da amostra tinha uma renda mensal per capita de um sa- lário-mínimo ou menos e completou até 4 anos de escolaridade. Houve predomínio de pessoas do proletariado não típico (43\%) seguido por pequena burguesia tradicional (19\%) e proletariado típico (18\%).

Foram estudados hábitos alimentares, número de refeições diárias, uso de açúcar, gorduras e a frequiência de consumo de alimentos específicos. A maior parte da amostra consumia pão e arroz, no mínimo, duas vezes por dia, enquanto que feijão e carne foram consumidos pelo menos duas vezes por dia por quase metade da amostra.

Com relação às atividades físicas das pessoas que executaram algum trabalho remunerado, mais da metade não realizava nenhum esforço físico no trabalho. Apenas 32\% fizeram algum exercício físico no lazer durante o último ano.

Quanto à ocorrência de morbidade, hipertensão arterial foi referida por $22 \%$ dos entrevistados e diabetes por $5 \%$. Trinta e oito por cento das pessoas referiram que sua mãe era obesa, enquanto que $20 \%$ relataram obesidade no pai.

A prevalência de obesidade na população estudada foi de $21 \%$, enquanto que quase $40 \%$ da amostra apresentou sobrepeso. A Tabela 1 mostra que a prevalência de obesidade é significativamente mais elevada em mulheres do que em homens (25\% e 15\%, respectivamente). Por outro lado, a proporção de obesidade aumenta marcadamente com a idade, sendo cerca de quatro vezes mais elevada após os 40 anos do que no grupo com idade entre 20 e 29 anos.

Observou-se uma associação inversa entre obesidade e nível de escolaridade, ou seja, as pessoas 
com maior escolaridade foram significativamente menos obesas. Apesar deste achado, não se encontrou associação entre obesidade e outras variáveis socioeconômicas, como renda familiar e classe social, exceto o subproletariado, que mostrou um risco de obesidade quase duas vezes maior que a burquesia e nova pequena burguesia.

Mulheres que tiveram um filho apresentaram risco de obesidade quase duas vezes maior do que nulíparas, e este risco chegou a cerca de três naquelas que tiveram três ou mais filhos.
As prevalências de obesidade (Tabela 2) também foram maiores entre as pessoas que não tiveram trabalho remunerado no mês anterior à entrevista, nem realizaram exercício físico no último ano.

Hipertensos e diabéticos apresentaram um risco 2,6 vezes maior de serem obesos do que aqueles que não referiram estas patologias. Com relação ao fumo, observou-se maiores prevalências de obesidade entre não fumantes.

Quanto a fatores familiares, as pessoas que referiram obesidade da mãe e do pai, apresentaram um

Tabela 1 - Prevalência de obesidade e razão de prevalência (RP) com intervalo de confiança (IC) de variáveis demográficas e socioeconômicas ( $\mathrm{n}=1.035)$.

Table1 - Prevalence of obesity and prevalence ratio (RP) with $95 \%$ confidence intervals (Cl) by demographic and socioeconomic variables $(n=1,035)$.

\begin{tabular}{|c|c|c|c|}
\hline Variável & $\begin{array}{c}\text { Prevalência } \\
\text { O besidade } \%\end{array}$ & $\mathrm{RP}(\mathrm{IC})$ & $\mathrm{N}$ \\
\hline \multicolumn{4}{|l|}{ Sexo } \\
\hline Masculino & 15 & 1,00 & 455 \\
\hline Feminino & 25 & $1,73(1,33$ a 2,24$)$ & 580 \\
\hline \multicolumn{4}{|l|}{ Idade } \\
\hline $20-29$ anos & 7 & 1,00 & 262 \\
\hline $30-39$ anos & 14 & $1,89(1,11$ a 3,21$)$ & 256 \\
\hline $40-49$ anos & 31 & $4,11(2,54$ a 6,65$)$ & 197 \\
\hline $50-59$ anos & 29 & $3,87(2,38$ a 6,31$)$ & 182 \\
\hline $60-69$ anos & 34 & $4,48(2,74$ a 7,32$)$ & 138 \\
\hline \multicolumn{4}{|l|}{ Cor } \\
\hline Branca & 21 & 1,00 & 881 \\
\hline Preta & 22 & $1,06(0,76$ a 1,47$)$ & 148 \\
\hline \multicolumn{4}{|l|}{ Situação conjugal } \\
\hline Sem companheiro & 18 & 1,00 & 701 \\
\hline Com companheiro & 22 & $1,28(0,98$ a 1,68$)$ & 334 \\
\hline \multicolumn{4}{|l|}{ Paridade* } \\
\hline Nulípara & 13 & 1,00 & 132 \\
\hline 1 filho & 22 & $1,74(1,00$ a 3,02$)$ & 125 \\
\hline 2 filhos & 23 & $1,78(1,04$ a 3,04$)$ & 144 \\
\hline 3 ou mais filhos & 38 & $2,95(1,82$ a 4,77$)$ & 179 \\
\hline \multicolumn{4}{|l|}{ Anos de escolaridade } \\
\hline 9 ou mais & 14 & 1,00 & 335 \\
\hline $5-8$ & 19 & $1,37(0,97$ a 1,94$)$ & 346 \\
\hline $1-4$ & 27 & $2,01(1,44$ a 2,80$)$ & 260 \\
\hline Nenhum & 37 & $2,71(1,84$ a 3,99$)$ & 81 \\
\hline \multicolumn{4}{|l|}{ Renda familiar mensal } \\
\hline $4^{\circ}$ quartil & 21 & 1,00 & 247 \\
\hline $3^{\circ}$ quartil & 17 & $0,78(0,54$ a 1,13$)$ & 246 \\
\hline $2^{\circ}$ quartil & 22 & $1,06(0,76$ a 1,48$)$ & 246 \\
\hline $1^{\circ}$ quartil & 22 & $1,07(0,77$ a 1,50$)$ & 247 \\
\hline \multicolumn{4}{|l|}{ Classe social (ABIPEME) } \\
\hline Classe A e B & 19 & 1,00 & 170 \\
\hline Classe C & 18 & $0,93(0,62$ a 1,39$)$ & 257 \\
\hline Classe D & 21 & $1,16(0,80$ a 1,67$)$ & 308 \\
\hline Classe $\mathrm{E}$ & 23 & $1,19(0,82$ a 1,74$)$ & 257 \\
\hline \multicolumn{4}{|l|}{ Classe social (BRONFMAN)** } \\
\hline Burguesia e NPB & 15 & 1,00 & 127 \\
\hline Pequena burguesia tradicional & 21 & $1,41(0,85$ a 2,35$)$ & 180 \\
\hline Proletariado não típico & 22 & $1,45(0,92$ a 2,28$)$ & 406 \\
\hline Proletariado típico & 20 & $1,31(0,78$ a 2,19$)$ & 167 \\
\hline Subproletariado & 28 & $1,93(1,07$ a 3,50$)$ & 55 \\
\hline
\end{tabular}

Algumas das variáveis acima apresentaram até $4,7 \%$ de respostas ignoradas.

$* \mathrm{n}=580$.

** Variável composta por escolaridade, renda e ocupação do chefe da família; apresentou 9,7\% de respostas ignoradas. 
risco de obesidade quase duas vezes maior que aqueles cujos pais não eram, segundo sua opinião, obesos (Tabela 2).

A análise estratificada de diferentes variáveis com obesidade, conforme o sexo, mostrou importantes diferenças entre os fatores de risco para obesidade. A Figura 2 apresenta a evolução da obesidade entre homens e mulheres de acordo com a idade. Pode-se notar que as prevalências de obesidade são semelhantes nos dois sexos até os 40 anos, mas após esta idade as mulheres passam a apresentar prevalências duas vezes mais elevadas do que os homens.

As Tabelas 3 e 4 mostram as associações das variáveis demográficas e socioeconômicas com a obesidade em homens e mulheres, respectivamente. Ob- serva-se relação direta de variáveis socioeconômicas com obesidade em homens (Tabela 3) e inversa com as mulheres (Tabela 4). A cor da pele dos entrevistados não mostrou diferença significativa na ocorrência de obesidade em homens e mulheres, porém os homens que viviam com companheiras apresentaram um risco de obesidade duas vezes maior que aqueles que viviam sozinhos, desaparecendo este efeito na análise ajustada.

A obesidade de um dos pais mostrou uma chance $50 \%$ maior de ser obeso do que aqueles entrevistados, tanto homens como mulheres, que referiram que nenhum dos pais era obeso (Tabelas 5 e 6 ).

Ainda na análise ajustada, observou-se que mulheres hipertensas, diabéticas e sem hábito de taba-

Tabela 2 - Prevalência de obesidade e razão de prevalência (RP) com intervalo de confiança (IC) conforme atividade física, morbidades referidas e variáveis relacionadas com alimentação $(n=1.035)$.

Table 2 - Prevalence of obesity and prevalence ratio (RP) with 95\% confidence intervals (CI) by physical activity, morbidity and feeding habits $(n=1,035)$.

\begin{tabular}{|c|c|c|c|}
\hline Variável & $\begin{array}{c}\text { Prevalência } \\
\text { O besidade } \%\end{array}$ & $\mathrm{RP}(\mathrm{IC})$ & $\mathrm{N}$ \\
\hline \multicolumn{4}{|l|}{ Trabalho remunerado } \\
\hline Sim & 17 & 1,00 & 625 \\
\hline Não & 26 & $1,50(1,18$ a 1,90$)$ & 408 \\
\hline \multicolumn{4}{|l|}{ Exercício físico } \\
\hline Sim & 13 & 1,00 & 314 \\
\hline $\mathrm{N}$ ão & 24 & $1,87(1,38$ a 2,54$)$ & 707 \\
\hline \multicolumn{4}{|c|}{$\mathrm{N}$ atureza da atividade } \\
\hline Pesada & 17 & 1,00 & 140 \\
\hline Moderada & 18 & $1,07(0,71$ a 1,62$)$ & 422 \\
\hline Leve & 24 & $1,43(0,96$ a 2,13$)$ & 471 \\
\hline \multicolumn{4}{|c|}{ Herança de obesidade } \\
\hline N enhum & 16 & 1,00 & 539 \\
\hline Somente pai & 24 & $1,52(0,99$ a 2,32$)$ & 83 \\
\hline Somente mãe & 24 & $1,52(1,14$ a 2,02$)$ & 265 \\
\hline Ambos & 31 & $1,93(1,38$ a 2,70$)$ & 114 \\
\hline \multicolumn{4}{|l|}{ Hipertensão arterial } \\
\hline Não & 15 & 1,00 & 786 \\
\hline Sim & 40 & $2,64(2,10$ a 3,31$)$ & 226 \\
\hline \multicolumn{4}{|l|}{ Diabetes mellitus } \\
\hline Não & 19 & 1,00 & 949 \\
\hline Sim & 51 & $2,62(1,95$ a 3,51$)$ & 53 \\
\hline \multicolumn{4}{|l|}{ Tabagismo } \\
\hline Fumante & 14 & 1,00 & 318 \\
\hline $\mathrm{N}$ ão fumante & 24 & $1,67(1,23$ a 2,28$)$ & 584 \\
\hline Ex-fumante & 23 & $1,63(1,08$ a 2,47$)$ & 130 \\
\hline \multicolumn{4}{|l|}{ Consumo de álcool } \\
\hline Sim & 16 & 1,00 & 606 \\
\hline Não & 28 & $1,83(1,44$ a 2,32$)$ & 428 \\
\hline \multicolumn{4}{|c|}{$\mathrm{N}^{\circ}$ de refeicões diárias } \\
\hline Até 3 & 23 & 1,00 & 504 \\
\hline Mais de 3 & 18 & $0,78(0,62$ a 1,00$)$ & 525 \\
\hline \multicolumn{4}{|l|}{ Consumo de óleo } \\
\hline A té 1 lata & 21 & 1,00 & 456 \\
\hline M ais de 1 lata & 22 & $1,04(0,82$ a 1,32$)$ & 548 \\
\hline \multicolumn{4}{|l|}{ Consumo de açúcar } \\
\hline Até $1,5 \mathrm{Kg}$ & 19 & 1,00 & 412 \\
\hline$M$ ais de $1,5 \mathrm{Kg}$ & 23 & $1,25(0,97$ a 1,61$)$ & 585 \\
\hline
\end{tabular}

Algumas das variáveis acima apresentaram até $3,7 \%$ de respostas ignoradas. 


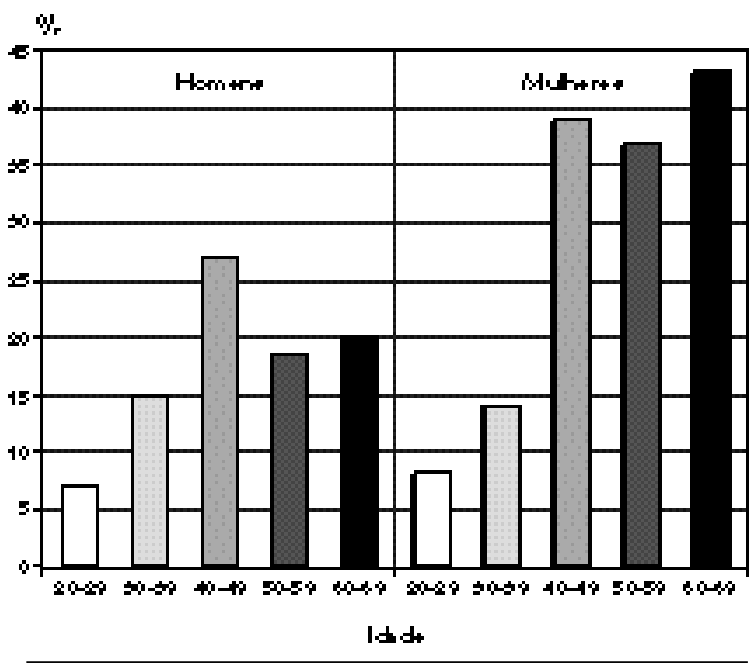

Figura 2 - Prevalência de obesidade segundo gênero e idade. Figure 2 - Prevalence of obesity gender and age.

gismo foram mais obesas (Tabela 6), enquanto que para os homens apenas a hipertensão manteve-se associada significativamente com a obesidade (Tabela 5). O número de refeições diárias mostrou uma relação inversa com obesidade. Enquanto que para as mulheres essa relação foi estatisticamente significativa (Tabela 6), para os homens esteve no limiar da significância (Tabela 5). Com relação às variáveis de atividade física, somente entre as mulheres o exercício físico no lazer, realizado no ano anterior, manteve-se significativo na análise ajustada.

\section{DISCU SSÃO}

Considerando a metodologia utilizada e o percentual de perdas e recusas, este estudo é representativo da população adulta, de 20 a 69 anos de idade, residente na zona urbana do Município de Pelotas.

A prevalência de obesidade esteve um ponto percentual acima daquela encontrada nesse mesmo município, em $1992^{21}$. Os resultados da $\mathrm{PNSN}^{8}$ mostraram que, na região Sul, em pessoas com 18 anos ou mais, as prevalências de obesidade e sobrepeso foram $13 \%$ e $27 \%$, respectivamente. Resultados desta mesma pesquisa apresentados em outro trabalho ${ }^{2}$, onde a população-alvo foram pessoas com 20 anos ou mais, mostraram prevalências maiores de sobrepeso e obesidade para o País como um todo. Tam-

Tabela 3 - Prevalências de obesidade de acordo com variáves demográficas e socioeconômicas em homens $(n=455)$.

Table 3 - Prevalence of obesity by demographic and socioeconomic variables in men $(n=455)$.

\begin{tabular}{|c|c|c|c|}
\hline Variável & $\begin{array}{c}\text { Prevalência } \\
\text { O besidade } \%\end{array}$ & $\begin{array}{c}\text { "O dds ratio" } \\
\text { Bruto }\end{array}$ & $\begin{array}{l}\text { "O dds ratio" } \\
\text { Ajustado* }\end{array}$ \\
\hline Renda familiar mensal & & & $0,79(0,62$ a 1,01$)$ \\
\hline $4^{\circ}$ quartil & 21 & 1,00 & \\
\hline $3^{\circ}$ quartil & 15 & $0,67(0,34$ a 1,33$)$ & \\
\hline $2^{\circ}$ quartil & 12 & $0,52(0,25$ a 1,05$)$ & \\
\hline $1^{\circ}$ quartil & 12 & $0,54(0,25$ a 1,13$)$ & \\
\hline Anos de escolaridade & & & $0,96(0,72$ a 1,29$)$ \\
\hline 9 ou mais & 14 & 1,00 & \\
\hline $5-8$ & 13 & $0,92(0,48$ a 1,75$)$ & \\
\hline $1-4$ & 19 & $1,41(0,73$ a 2,74$)$ & \\
\hline Nenhum & 14 & $0,97(0,31$ a 3,06$)$ & \\
\hline Classe social (ABIPEME) & & & $0,78(0,61$ a 1,00$)$ \\
\hline Classe $A$ e $B$ & 20 & 1,00 & \\
\hline Classe C & 18 & $0,87(0,41$ a 1,81$)$ & \\
\hline Classe D & 13 & $0,58(0,28$ a 1,21$)$ & \\
\hline Classe $\mathrm{E}$ & 12 & $0,56(0,26$ a 1,23$)$ & \\
\hline Classe social (BRONFMAN) & & & $0,89(0,69$ a 1,16$)$ \\
\hline Burguesia e NPB & 17 & 1,00 & \\
\hline Pequena burguesia tradicional & 18 & $1,04(0,42$ a 2,58$)$ & \\
\hline Proletariado não típico & 15 & $0,83(0,37$ a 1,87$)$ & \\
\hline Proletariado típico & 8 & $0,42(0,14$ a 1,25$)$ & \\
\hline Subproletariado & 23 & $1,41(0,41$ a 4,80$)$ & \\
\hline Cor & & & $0,94(0,39$ a 2,29$)$ \\
\hline Branca & 16 & 1,00 & \\
\hline Preta & 11 & $0,69(0,30$ a 1,59$)$ & \\
\hline Situação conjugal & & & $1,67(0,79$ a 3,55$)$ \\
\hline Sem companheiro & 8 & 1,00 & \\
\hline Com companheiro & 17 & $2,19(1,08$ a 4,44$)$ & \\
\hline
\end{tabular}

* Análise ajustada hierarquicamente para idade e variáveis socioeconômicas. 
bém deve-se considerar que na $\mathrm{PNSN}^{8}$ as freqüências de sobrepeso e obesidade foram menores a partir dos 55 anos de idade.

As prevalências de obesidade foram maiores para as mulheres e aumentaram de acordo com a idade, discordando dos resultados da $\mathrm{PNSN}^{8}$. Porém, chamou atenção que a prevalência entre os homens apresentou uma relativa estabilização a partir dos 40 anos, enquanto que para as mulheres as prevalências dobraram, a partir desta idade.

Com relação à paridade, observou-se que a associação positiva desaparece quando variáveis socioeconômicas e idade são ajustadas, concordando com resultados de outros estudos ${ }^{6}$. A situação conjugal mostrou associação com obesidade somente entre os homens, da mesma forma que em estudo realizado por Sobal ${ }^{27}$. Entretanto as variáveis socioeconômicas confundiram esta associação, tendo o efeito desaparecido na análise ajustada.
Estudos sobre dieta e obesidade descrevem correlação positiva com consumo de gordura, especialmente saturada, em mulheres de meia-idade ${ }^{23}$. Também um menor número de refeições está associado com excesso de peso e elevação na concentração de colesterol e glicemia ${ }^{5}$. No presente trabalho não se encontrou associação entre obesidade e consumo de gorduras, e o efeito protetor do maior número de refeições manteve-se para as mulheres mesmo após a análise ajustada, enquanto que para os homens permaneceu com significância estatística próxima ao limite.

Com relação ao consumo de álcool, observou-se uma associação inversa com obesidade nas mulheres. Entretanto este efeito desapareceu na análise ajustada. Estes resultados concordam com a revisão de Hellerstedt e col. ${ }^{12}$, onde a maioria dos estudos sugere que consumidores de álcool pesam menos quando comparados aos não consumidores com si-

Tabela 4 - Prevalências de obesidade de acordo com variáveis demográficas e socioeconômicas em mulheres ( $n=580$ ).

Table 4 - Prevalence of obesity by demographic and socioeconomic variables in women $(n=580)$.

\begin{tabular}{|c|c|c|c|}
\hline Variável & $\begin{array}{c}\text { Prevalência } \\
\text { O besidade } \%\end{array}$ & $\begin{array}{c}\text { "O dds ratio" } \\
\text { Bruto }\end{array}$ & $\begin{array}{l}\text { "O dds ratio" } \\
\text { Ajustado* }\end{array}$ \\
\hline Renda familiar mensal & & & $1,20(1,00$ a 1,43$)$ \\
\hline $4^{\circ}$ quartil & 22 & 1,00 & \\
\hline $3^{\circ}$ quartil & 20 & $0,89(0,50$ a 1,59$)$ & \\
\hline $2^{\circ}$ quartil & 30 & $1,59(0,92$ a 2,74$)$ & \\
\hline $1^{\circ}$ quartil & 29 & $1,45(0,86$ a 2,47$)$ & \\
\hline Anos de escolaridade & & & $1,47(1,18$ a 1,84$)$ \\
\hline 9 ou mais & 13 & 1,00 & \\
\hline $5-8$ & 23 & $1,96(1,16$ a 3,40$)$ & \\
\hline $1-4$ & 33 & $3,27(1,91$ a 5,62$)$ & \\
\hline Nenhum & 50 & $6,48(3,26$ a 12,89$)$ & \\
\hline Classe social (ABIPEME) & & & $1,30(1,06$ a 1,59$)$ \\
\hline Classe A e B & 19 & 1,00 & \\
\hline Classe C & 18 & $0,98(0,50$ a 1,91$)$ & \\
\hline Classe D & 28 & $1,65(0,89$ a 3,04$)$ & \\
\hline Classe $\mathrm{E}$ & 32 & $1,97(1,04$ a 3,71$)$ & \\
\hline Classe social (BRONFMAN) & & & $1,27(1,04$ a 1,56$)$ \\
\hline Burguesia e NPB & 13 & 1,00 & \\
\hline Pequena burguesia tradicional & 23 & $1,93(0,85$ a 4,38$)$ & \\
\hline Proletariado não típico & 27 & $2,42(1,18$ a 4,94$)$ & \\
\hline Proletariado típico & 29 & $2,70(1,21$ a 6,03$)$ & \\
\hline Subproletariado & 30 & $2,83(1,04$ a 7,66$)$ & \\
\hline Cor & & & $1,16(0,66$ a 2,05$)$ \\
\hline Branca & 25 & 1,00 & \\
\hline Preta & 29 & $1,26(0,76$ a 2,10$)$ & \\
\hline Paridade & & & $1,14(0,92$ a 1,40$)$ \\
\hline Nulípara & 13 & 1,00 & \\
\hline 1 filho & 22 & $1,95(1,01$ a 3,78$)$ & \\
\hline 2 filhos & 23 & $2,01(1,06$ a 3,82$)$ & \\
\hline 3 ou mais filhos & 38 & $4,14(2,29$ a 7,49$)$ & \\
\hline Situação conjugal & & & $1,36(0,90$ a 2,14$)$ \\
\hline Sem companheiro & 23 & 1,00 & \\
\hline Com companheiro & 27 & $1,26(0,85$ a 1,87$)$ & \\
\hline
\end{tabular}

* Análise ajustada hierarquicamente para idade e variáveis socioeconômicas. 
milar consumo de calorias, com dados inconsistentes entre os sexos e sem que se possa estabelecer causalidade. De acordo com esses autores, o papel do álcool no mecanismo de determinação da adiposidade não está bem determinado.

A transmissão familiar de obesidade é bem conhecida ${ }^{15}$, acreditando-se que pode ser tanto por fatores genéticos, como por estilo de vida ${ }^{22}$. A ocorrência de obesidade dos pais levou a um risco de 1,3 entre os homens e 1,2 entre as mulheres para cada categoria da variável, chegando a um risco duas vezes maior para os entrevistados que informaram que tanto a mãe como o pai eram obesos. Mesmo controlando para variáveis demográficas e socioeconômicas esta associação manteve-se inalterada.
Em concordância com os resultados da $\mathrm{PNSN}^{26}$, a obesidade esteve positivamente associada com renda, somente entre os homens. Entretanto os presentes resultados indicam uma associação inversa entre nível de escolaridade e obesidade nas mulheres, sugerindo uma tendência em direção ao que ocorre em países desenvolvidos ${ }^{28}$.

Ao concluir a análise ajustada para possíveis fatores de confusão, convém notar que a relação de algumas variáveis estudadas, como morbidades referidas, consumo de açúcar e até mesmo exercício físico, com a ocorrência de obesidade, é de difícil interpretação em um estudo de tipo transversal, uma vez que não pode ser afastada a possibilidade de causalidade reversa. Mesmo assim, a plausibilidade bi-

Tabela 5 - Prevalências de obesidade de acordo com morbidades referidas e variáveis de alimentação e de atividade física em homens $(n=455)$.

Table 5 - Prevalence of obesity by morbidity, physical activity and feeding habits in men $(n=455)$

\begin{tabular}{|c|c|c|c|}
\hline Variável & $\begin{array}{c}\text { Prevalência } \\
\text { O besidade } \%\end{array}$ & $\begin{array}{c}\text { "O dds ratio" } \\
\text { Bruto }\end{array}$ & $\begin{array}{l}\text { "O dds ratio" } \\
\text { Ajustado* }\end{array}$ \\
\hline Herança de obesidade & & & $1,58(1,10$ a 2,29$)$ \\
\hline Nenhum & 13 & 1,00 & \\
\hline Pai/M ãe & 16 & $1,37(0,76$ a 2,47$)$ & \\
\hline Ambos & 21 & $2,40(1,14$ a 5,06$)$ & \\
\hline Diabetes mellitus & & & $2,71(0,97$ a 7,62$)$ \\
\hline Não & 13 & 1,00 & \\
\hline Sim & 42 & $4,56(1,76$ a 11,79$)$ & \\
\hline Hipertensão arterial & & & $2,85(1,53$ a 5,29$)$ \\
\hline Não & 11 & 1,00 & \\
\hline Sim & 31 & $3,44(1,95$ a 6,07$)$ & \\
\hline Tabagismo & & & $0,97(0,70$ a 1,35$)$ \\
\hline Fumante & 14 & 1,00 & \\
\hline $\mathrm{N}$ ão fumante & 21 & $1,75(0,88$ a 3,48$)$ & \\
\hline Ex-fumante & 14 & $1,00(0,55$ a 1,82$)$ & \\
\hline Consumo de álcool & & & $1,25(0,66$ a 2,35$)$ \\
\hline Sim & 14 & 1,00 & \\
\hline Não & 17 & $1,28(0,72$ a 2,29$)$ & \\
\hline Consumo de açúcar & & & $1,08(0,60$ a 1,94$)$ \\
\hline Até $1,5 \mathrm{Kg}$ & 15 & 1,00 & \\
\hline M ais de $1,5 \mathrm{Kg}$ & 15 & $0,98(0,58$ a 1,66$)$ & \\
\hline Consumo de óleo & & & $1,31(0,72$ a 2,37$)$ \\
\hline Até 1 lata & 14 & 1,00 & \\
\hline Mais de 1 lata & 16 & $1,23(0,73$ a 2,08$)$ & \\
\hline $\mathrm{N}^{0}$ de refeições diárias & & & $0,58(0,33$ a 1,04$)$ \\
\hline Até 3 & 18 & 1,00 & \\
\hline 4 a 6 & 12 & $0,60(0,35$ a 1,02$)$ & \\
\hline Trabalho remunerado & & & $0,85(0,43$ a 1,71$)$ \\
\hline Sim & 15 & 1,00 & \\
\hline Não & 16 & $1,13(0,61$ a 2,08$)$ & \\
\hline Exercício físico & & & $1,15(0,75$ a 1,76$)$ \\
\hline Sim & 13 & 1,00 & \\
\hline Não & 17 & $1,38(0,80$ a 2,37$)$ & \\
\hline Natureza da atividade & & & $1,19(0,65$ a 2,18$)$ \\
\hline Pesada & 14 & 1,00 & \\
\hline Moderada & 12 & $0,88(0,37$ a 2,10$)$ & \\
\hline Leve & 18 & $1,39(0,61$ a 3,17$)$ & \\
\hline
\end{tabular}

* As variáveis foram ajustadas hierarquicamente, incluindo idade, variáveis socioeconômicas, outras variáveis demográficas, morbidades referidas e variáveis de alimentação e de atividade física. 
ológica e a magnitude de efeito de variáveis como escolaridade, obesidade dos pais e o tabagismo para as mulheres sugerem que possam ser interpretadas como possíveis fatores causais. Quanto ao número de refeições acredita-se que poderia ser considerado um marcador de risco uma vez que a concentração do consumo em uma única refeição diária parece estar associada com mais rápida formação de gordura ${ }^{5}$.

Ainda com relação às variáveis socioeconômicas, tem sido sugerido que estas podem ser influenciadas pela obesidade, ou seja, que a obesidade pode precedê-las ${ }^{29}$. Gortmaker e col. ${ }^{11}$, em um estudo lon- gitudinal com 10.000 pessoas de 16 a 24 anos, encontraram, após sete anos de acompanhamento, que mulheres com excesso de peso casavam menos, tinham rendas mais baixas e completavam menos anos na escola.

Os presentes resultados indicam que os determinantes da obesidade são diferentes para homens e mulheres e esta perspectiva pode ser considerada em futuros trabalhos. O fato de que a prevalência de obesidade nas mulheres dobrou a partir de 40 anos, propõe a investigação de outros possíveis determinantes.

Tabela 6 - Prevalências de obesidade de acordo com morbidades referidas e variáveis de alimentação e de atividade física em mulheres $(n=580)$.

Table 6 - Prevalence of obesity by morbidity, physical activity and feeding habits in women $(n=580)$.

\begin{tabular}{|c|c|c|c|}
\hline Variável & $\begin{array}{c}\text { Prevalência } \\
\text { O besidade } \%\end{array}$ & $\begin{array}{c}\text { "O dds ratio" } \\
\text { Bruto } \\
\end{array}$ & $\begin{array}{l}\text { "O dds ratio" } \\
\text { Ajustado* }\end{array}$ \\
\hline Herança de obesidade & & & $1,44(1,08$ a 1,92$)$ \\
\hline Nenhum & 20 & 1,00 & \\
\hline Pai/Mãe & 29 & $1,56(1,04$ a 2,34$)$ & \\
\hline Ambos & 34 & $2,03(1,14$ a 3,62$)$ & \\
\hline Diabetes mellitus & & & $2,25(1,01$ a 5,02$)$ \\
\hline Não & 23 & 1,00 & \\
\hline Sim & 56 & $4,18(2,06$ a 8,46$)$ & \\
\hline Hipertensão arterial & & & $2,33(1,46$ a 3,70$)$ \\
\hline Não & 18 & 1,00 & \\
\hline Sim & 46 & $3,71(2,47$ a 5,57$)$ & \\
\hline Tabagismo & & & $1,56(1,19$ a 2,04$)$ \\
\hline Fumante & 15 & 1,00 & \\
\hline $\mathrm{N}$ ão fumante & 26 & $2,03(0,92$ a 4,48$)$ & \\
\hline Ex-fumante & 29 & $2,38(1,45$ a 3,90$)$ & \\
\hline Consumo de álcool & & & $1,29(0,82$ a 2,03$)$ \\
\hline Sim & 17 & 1,00 & \\
\hline Não & 31 & $2,13(1,44$ a 3,17$)$ & \\
\hline Consumo de açúcar & & & $1,23(0,77$ a 1,95$)$ \\
\hline Até $1,5 \mathrm{Kg}$ & 20 & 1,00 & \\
\hline M ais de $1,5 \mathrm{Kg}$ & 29 & $1,58(1,06$ a 2,35$)$ & \\
\hline Consumo de óleo & & & $0,81(0,52$ a 1,26$)$ \\
\hline Até 1 lata & 25 & 1,00 & \\
\hline Mais de 1 lata & 25 & $1,04(0,71$ a 1,51$)$ & \\
\hline $\mathrm{N}^{\circ}$ de refeições diárias & & & $0,56(0,37$ a 0,86$)$ \\
\hline A té 3 & 29 & 1,00 & \\
\hline 4 a 6 & 22 & $0,72(0,49$ a 1,04$)$ & \\
\hline Trabalho remunerado & & & $0,86(0,55$ a 1,35$)$ \\
\hline Sim & 21 & 1,00 & \\
\hline Não & 29 & $1,49(1,02$ a 2,19$)$ & \\
\hline Exercício físico & & & $1,77(0,99$ a 3,17$)$ \\
\hline Sim & 13 & 1,00 & \\
\hline Não & 29 & $2,55(1,52$ a 4,27$)$ & \\
\hline Natureza da atividade & & & $1,20(0,87$ a 1,67$)$ \\
\hline Pesada & 20 & 1,00 & \\
\hline M oderada & 23 & $1,20(0,64$ a 2,24$)$ & \\
\hline Leve & 29 & $1,66(0,90$ a 3,04$)$ & \\
\hline
\end{tabular}

*As variáveis foram ajustadas hierarquicamente, incluindo idade, variáveis socioeconômicas, outras variáveis demográficas, morbidades referidas e variáveis de alimentação e de atividade física. 


\section{REFERÊN CIAS BIBLIO GRÁFICAS}

1. ANJOS, L.A. Índice de massa corporal (massa corporal.estatura-2 ${ }^{2}$ como indicador do estado nutricional de adultos: revisão de literatura. Rev. Saúde Pública, 26: 431-6, 1992.

2. ANJOS, L.A. Valores antropométricos da população adulta brasileira: resultados da pesquisa nacional sobre saúde e nutrição. Rio de Janeiro, 1993. [Tese de Livre-Docência Departamento de Nutrição Social da UFF].

3. BARROS, F.C. \& VICTORA, C.G. Epidemiologia da saúde infantil. São Paulo, Ed Hucitec-Unicef, 1994.

4. BAUMGARTNER, R.N.; HEYMSFIELD, S.B.; ROCHE, A.F. Human body composition and the epidemiology of chronic disease. Obesity Res., 3: 73-95, 1995.

5. BRAY, G. An approach to the classification and evaluation of obesity. In: Björntorp, P. \& Brodoff, B.N. Obesity. New York, JB Lippincott Company, 1992. p. 294-308.

6. BROWN, J.E.; KAYE, S.A.; FOLSOM, A.R. Parity-related weight change in women. Intern. J. Obesity, 16: 62731,1992 .

7. FUNDAÇÃO IBGE. Censo demográfico: resultados do universo relativos às características da população e dos domicílios - Rio Grande do Sul. Rio de Janeiro, 1991. t.1,n.20, p.1-688.

8. COITINHO, D.C.; LEÃO, M.M.; RECINE, E.; SICHIERI, R. Condições nutricionais da população brasileira: adultos e idosos. Brasília, 1991.(Pesquisa Nacional sobre Saúde e Nutrição, MS/INAN).

9. DUNCAN, B.B. As desigualdades sociais na distribuição de fatores de risco para doenças não transmissíveis. Porto Alegre, 1991. [Tese de Doutorado - Universidade Federal do Rio Grande do Sul].

10. GARFINKELL, L. Overweight and cancer. Ann. Intern. Med., 103: 1034-6, 1985.

11. GORTMAKER, S.L.; MUST, A.; PERRIN, J.M.; SOBOL, A.M.; DIETZ, W.H. Social and economic consequences of overweight in adolescence and young adulthood. N. Engl. J. Med., 329: 1008-12, 1993.

12. HELLERSTEDT, W.L.; JEFFERY, R.W.; MURRAY, D.M. The association between alcohol intake and adiposity in the general population. Am. J. Epidemiol., 132: 594-611,1990.

13. KUCZMARSKI, R.J. Prevalence of overweight and weight gain in the United States. Am. J. Clin. Nutr., 55: 495S-502S, 1992.

14. KUSKOWSKA-WOLK, A. \& BERGSTRÖM, R. Trends in body mass index and prevalence of obesity in Swedish women 1980-89. J. Epidemiol. Comm. Health, 47: 195-9, 1993.

15. LOCARD, E.; MAMELLE, N.; BILLETTE, A.; MIGINIAC, M.; MUNOZ, F.; REY, S. Risk factors of obesity in a five year old population. Parental versus environmental factors. Int. J. Obesity, 16: 721-9, 1992.
16. LOHMAN, T.G.; ROCHE, A.F.; MARTORELL, R. Anthropometric standardization reference manual. Champaign, Ill. Human Kinetics Books, 1988.

17. LOMBARDI, C.; BRONFMAN, M.; FACCHINI, L.A.; VICTORA, C.G.; BARROS, F.C.; BÉRIA, J.U.; TEIXEIRA, A.M.B. Operacionalização do conceito de classe social em estudos epidemiológicos. Rev. de Saúde Pública, 22: 253-65, 1988.

18. MANSON, J.E.; COLDITZ, G.A.; STAMPFER, M.J.; WILLETT, W.C.; ROSNER, B.; MONSON, R.R.; SPEIZER, F.E.; HENNEKENS, C.H. A prospective study of obesity and risk of coronary heart disease in women. $N$. Engl. J. Med., 322: 882-9, 1990.

19. MYKKÄNEN, L.; LAAKSO, M.; PYÖRÄLÄ, K. Association of obesity and distribution of obesity with glucose tolerance and cardiovascular risk factors in the elderly. Int. $J$. Obesity, 16: 695-704, 1992.

20. PI-SUNYER, F.X. Health implications of obesity. Am. J. Clin. Nutr., 53 (Suppl):1595-603, 1991.

21. PICCINI, R.X. Hipertensão arterial sistêmica em Pelotas, RS: prevalência, fatores de risco e manejo. Pelotas, 1993. [Dissertação de Mestrado - Universidade Federal de Pelotas].

22. RAVUSSIN, E. \& SWINBURN, B.A. Pathophysiology of obesity. Lancet, 340: 404-8, 1992.

23. ROMIEU, I.; WILLETT, W.C.; STAMPFER, M.J.; COLDITZ, G.A.; SAMPSON, L.; ROSNER, B.; HENNEKENS, C.H.; SPEIZER, F.E. Energy intake and other determinants of relative weight. Am. J. Clin. Nutr., 47: 406-12, 1988.

24. RUTTER, M. Pesquisa de mercado. São Paulo, Ed. Ática, 1988

25. RYAN, A.S.; ROCHE, A.F.; WELLENS, R.; GUO, S. Relationship of blood pressure to fatness and fat patterning in mexican american adults from the hispanic health and nutrition examination survey (HHANES, 1982-1984). Coll. Antropol., 18: 89-99, 1994.

26. SICHIERI, R.; COITINHO, D.C.; LEÃO, M.M.; RECINE. E.; EVERHART, J.E. High temporal, geographic, and income variation in body mass index among adults in Brazil. Am. J. Public Health, 84: 793-8, 1994.

27. SOBAL, J.; RAUSCHENBACH, B.S.; FRONGILLO, JR., E.A. Marital status, fatness and obesity. Soc. Sci. Med., 35: 915-23, 1992.

28. SOBAL, J. \& STUNKARD, A.J. Socioeconomic status and obesity: a review of the literature. Psych. Bull., 105: 260-75, 1989.

29. STUNKARD, A.J. \& SORENSEN, T.I.A. Obesity and socioeconomic status - a complex relation. N. Engl. J. Med., 329: 1036-7, 1993.

30. VAN ITALLIE, T.B. Health implications of overweight and obesity in the United States. Ann. Intern. Med., 103: 983-8, 1985.

31. WORLD HEALTH ORGANIZATION. Diet, nutrition, and the prevention of chronic diseases. Geneva, 1990. (WHO Technical Report Series 797). 